\title{
LAS OPERACIONES DE FUSIONES CON UNA SOCIEDAD CON PROPÓSITO ESPECIAL DE ADQUISICIÓN (SOCIEDAD SPAC)
}

\author{
MERGERS WITH A SPECIAL PURPOSE ACQUISITION COMPANY (SPAC)
}

\author{
Jorge L. Conde Granados ${ }^{1}$ \\ Universidad de San Martín de Porres \\ Lima- Perú. \\ https://orcid.org/0000-0001-6734-3 \\ jorgel@conde-granados.com
}

"Coming together is a beginning, keeping together is progress, working together is success".

Henry Ford

\section{RESUMEN}

Luego de casi dos (02) años de pandemia y gracias a la vacunación masiva, la reactivación económica tiene hoy un despegue mayor, haciendo que las empresas retomen sus proyectos entre los cuales se encuentran las operaciones de fusiones y adquisiciones. Pero, en los últimos tres (03) años el mercado internacional, empresarial y el legal se ha visto positivamente sorprendido en virtud a la incursión de un no tan nuevo modelo empresarial, y esta es la figura de la sociedad con propósito especial de adquisición (sociedad SPAC) sociedad que surgió en los años 80 y que en los últimos tres (03) años han sido masivamente utilizadas con el objetivo que una compañía privada pase a ser pública luego de una operación de fusión con SPAC. En este paper comentaremos un poco a cerca de este tema.

\section{PALABRAS CLAVES}

Fusión, adquisición, SPAC, sociedad, sociedad con propósito especial de adquisición, mercado, oferta pública de venta (OPV) u oferta pública inicial (IPO), operaciones de concentración, Mercado de Valores.

\section{ABSTRACT}

After almost two years since the outbreak of the pandemic and thanks to mass vaccination, economic reactivation has today more chances of success, hence companies are resuming their projects, including mergers and acquisitions. Furthermore, in the last three years, the international business and legal markets have been positively surprised by the increased use of a not-so-new business model: the Special Purpose Acquisition Company (SPAC), a type of company that first appeared in the 1980s. In the past three years, SPACs have been broadly used by private companies seeking to go public. This paper examines this matter.

\section{KEYWORDS}

Merger, acquisition, SPAC, company, Special Purpose Acquisition Company, market, initial public offering, concentration operations, Stock Market

1 Socio de la Consultora Conde \& Granados Asociados. Abogado. Magíster Magna Cum Laude Derecho Empresarial Universidad de Lima (UL). Reconocido como uno de los mejores gerentes legales de Perú por la Internacional LEGAL500. Miembro del grupo de expertos que dio el Anteproyecto de la Ley General de Sociedades peruano. Especialización en Project Finance por la Escuela de Negocios del Instituto de Empresa (IE) Madrid- España. Estudios de Posgrados en Argentina, EEUU y Chile. Docente Universidad de San Martín de Porres y en la Universidad Privada del Norte. 


\section{INTRODUCCIÓN}

Las operaciones de fusiones y adquisiciones a setiembre de 2021 en América Latina, han tocado un máximo de lo que fue hace 10 años por montos totales a los US\$105,000 millones según datos de Refinitv. Esto quiere decir, que hoy en día hemos regresado a niveles muy positivos de lo que fue a fines del 2011, en virtud a las reorganizaciones societarias, así como los emprendimientos empresariales y el nivel de cash Flow que tienes las empresas (y por qué no algunas startups) de algunos sectores favorecidos por este tema de la pandemia (alimentos, financiera, salud, protección, energía, entre otros), hacen que no solo miren hacia adentro sino también buscar oportunidades en el mercado para crecer a través de fusiones, o por un tema de estrategias decidieron una reorganización/desprenderse de su patrimonio con el objetivo de aliarse y/o no verse perjudicadas por la pandemia.

En esa búsqueda de proyectarse en el mercado internacional, hace importante que figuras no muy bien vistas en los años 80 y 90 , regresen a ser protagonistas, justamente con la idea de poder dejar de lado su actuación privada en las que se manejan para poder pasar al ámbito público a través justamente de esta reunión de patrimonios (fusión) con las Special Purpose Acquisition Company (SPAC Company) que ya intervienen en el mercado de valores pero que se constituyen estas a través de una operación conocida como Oferta Pública de Venta (OPV) u Oferta Pública Inicial (OPI) y una vez ya logradas, producir dicha fusión y en consecuencia dichas empresas privadas volverse públicas y listas para captar liquidez y continuar con sus operaciones, evitando el largo camino regulatorio para poder intervenir el mercado de valores. Entonces, que tienen en común operaciones como la de WeWork, Virgin Orbit y The Pegasus Europe ${ }^{2}$, en que todas ellas salieron al mercado a través de la figura SPAC.

Creemos que con la incursión de este tipo empresarial (algo antiguo pero muy utilizado hoy en día), no hace más que pensar que el derecho societario y el mundo de las fusiones y adquisiciones, no nos dejan de sorprender, que cuando se juntan pueden realizar acciones increíbles, algo parecido a traer los recuerdos del pasado no tan gratos, pero con el objetivo de poder aplicarlos $y$ reivindicarlos hoy, pero de manera positiva.

\section{2. ¿QUÉ SON LAS OPERACIONES DE FUSIÓN?}

Las operaciones de fusión de empresas, son aquellas operaciones de carácter corporativo (económica, financiera, legal), mediante el cual dos o más empresas deciden juntar sus patrimonios con el propósito de formar una compañía o mantenerse una en el mercado y a cambio los socios de las sociedad que intervienen en la operación de fusión por incorporación (nueva compañía) recibirán acciones y/o participaciones de la nueva sociedad, y para el caso de la fusión por absorción, los socios de la absorbida recibirán acciones y/o participaciones de la absorbente, produciéndose por tanto la extinción de las sociedades participantes en la fusión.

Hay algunas definiciones interesantes como:

La fusión como institución propia del derecho de personas jurídicas se caracteriza por implicar la unificación de dos o más personas jurídicas en una sola. Como ya dijimos, en el campo del derecho de las sociedades la fusión es una de las vías por las que se

2 WeWork, sube en la Bolsa tras fusión con una SPAC (Bow XAcquisition Corp) valorizado en US\$ 9,000MM, Virgin Orbit empresa que lanzo su primer viaje turístico al espacio se fusionó con una SPAC (Capital Hedosophia Holdings) valorizado en US\$1,500MM y Pegasus Europe obtuvo US\$ 606MM. 
instrumenta la concentración de empresas, obteniéndose la unión económica y jurídica de las respectivas sociedades, mientras que por los otros métodos de concentración antes expuestos se logra la unión económica, pero se mantienen las diversas personalidades jurídicas de las sociedades interesadas. Mediante la fusión, los patrimonios de dos o más sociedades que denominaremos sociedades fusionantes se unifican en un solo patrimonio $\mathrm{y}$, consecuentemente, en vez de coexistir varias personas jurídicas o sujetos de derecho existirá en lo sucesivo una sola persona jurídica o sujeto de derecho que llamaremos sociedad fusionaría. (Otaegui, 1981, p. 39)

En ese sentido, podemos afirmar que las operaciones de fusiones, son por excelencia mecanismos para reorganizar no solo la parte societaria, sino también para enfrentar nuevos desafíos en el ámbito del mercado sea geográfico y/o de producto en un determinado país, y como consecuencia de ello, generar una concentración empresarial que pueda o no atentar contra la libre competencia, por lo que en dicho análisis se tendría que optar por ver las normas en materia de control de fusiones en caso se encuentre regulado en aquel país en donde se esté desarrollando la operación de fusión.

Y, un poco para cerrar de manera general respecto a las operaciones de fusiones, vamos a traer a colación la regulación de carácter societario y en materia de defensa de la competencia (aunque en las operaciones de fusiones con SPAC no aplica) respectivamente:

\section{Ley Nº 26887 - Ley General de Sociedades}

\section{Artículo 344.- Concepto y formas de fusión}

Por la fusión dos a más sociedades se reúnen para formar una sola cumpliendo los requisitos prescritos por esta ley. Puede adoptar alguna de las siguientes formas:

1. La fusión de dos o más sociedades para constituir una nueva sociedad incorporante origina la extinción de la personalidad jurídica de las sociedades incorporadas y la transmisión en bloque, y a título universal de sus patrimonios a la nueva sociedad; o,

2. La absorción de una o más sociedades por otra sociedad existente origina la extinción de la personalidad jurídica de la sociedad o sociedades absorbidas. La sociedad absorbente asume, a título universal, y en bloque, los patrimonios de las absorbidas.

En ambos casos los socios o accionistas de las sociedades que se extinguen por la fusión reciben acciones o participaciones como accionistas o socios de la nueva sociedad o de la sociedad absorbente, en su caso.

\footnotetext{
Ley $\mathrm{N}^{\circ} 31112$ - Ley que establece el control previo de operaciones de concentración empresarial
}

\section{Artículo 5.- Operaciones de concentración empresarial}

5.1 Es todo acto u operación que implique una transferencia o cambio en el control de una empresa o parte de ella. Dichas concentraciones pueden producirse a consecuencia de las siguientes operaciones: 
a. Una fusión de dos o más agentes económicos, los cuales eran independientes antes de la operación, cualquiera que sea la forma de organización societaria de las entidades que se fusionan o de la entidad resultante de la fusión. (...)

Como vemos todo bien hasta aquí con relación a las fusiones propias, sin embargo, cuando se trata de realizar fusiones con una SPAC, aquí el tratamiento es diferente y es que se trata tanto de operaciones de fusiones propias como también de un tipo de fusión inversa (no como tal, pero al menos en su esencia), en donde tiene la misma connotación que las fusiones propias, pero al revés, esto es aquella fusión de carácter vertical en donde una sociedad filial absorbe a su matriz, es decir la sociedad absorbida (matriz) es titular de forma directa o indirecta de todas las acciones o participaciones de la sociedad absorbente (filial), la que en este caso la matriz se disuelve y extingue transmitiendo la totalidad de su patrimonio a la absorbente y produciendo las consecuencias jurídicas propias de una fusión por absorción, disolución sin liquidación y extinción de la sociedad absorbida.

Creemos que, con esta visión general, podemos ya dar un primer paso para ir avanzando en llegar a la integración de esta figura con la denominada Sociedad con Propósito Especial de Adquisición o Special Purpose Acquisition Company (en adelante "SPAC"), que será materia de análisis a continuación.

\section{3. ¿QUÉ ES LA SOCIEDAD CON PROPÓSITO ESPECIAL DE ADQUISICIÓN (SPAC)?.}

Luego de haber visto la figura de las operaciones de fusiones, los que nos toca es dar una visión general al igual que la primera sobre la figura de las SPAC, y ver cómo es su participación a nivel internacional.

Al respecto De Haro, José (2021) respecto al concepto de SPAC, nos dice que son "son entidades que no tienen un plan o propósito comercial específico y cuyo modelo de negocio suele estar centrado en participar en una fusión o adquisición con otras empresas" (p. 3).

Por su parte, Barón, Santiago (2021) define a las SPAC como "un tipo de compañías muy particulares, sin una actividad regular, como cualquier multinacional o pequeño negocio en el que puedas estar pensando. Son además un instrumento que se está generalizando en los mercados financieros" (p. 1).

Finalmente, España ha dado un salto en mayo del 2021, al publicar su Anteproyecto ${ }^{3}$ de Reforma de la Ley de Mercado de Valores y de los Servicios de Inversión español en donde proponen modificaciones a la Ley de Sociedades de Capital, justamente incorporando esta figura de las SPAC, en su nuevo modelo societario:

\section{Artículo 535 bis. Sociedad cotizada con propósito para la adquisición.}

1. Se entenderá por sociedad cotizada con propósito para la adquisición aquella que se constituya con el objeto de adquirir la totalidad o una participación en el capital de otra sociedad o sociedades cotizadas o no cotizadas, ya sea directa o indirectamente, a título de compraventa, fusión, escisión, aportación no dineraria, cesión global de activos y

3 Ver en: https://portal.mineco.gob.es/RecursosArticulo/mineco/ministerio/participacion_publica/audiencia/ficheros/ECO_ Tes_20210405_AP_LMV.pdf 
pasivos u otras operaciones análogas y cuyas únicas actividades hasta ese momento sean la oferta pública de valores inicial, la solicitud a admisión a negociación y las conducentes a la adquisición que, en su caso, sea aprobada por la Junta General de accionistas. (...)

Entonces, como podemos ver, la figura del SPAC no solo involucra un tema de financiamiento acudiendo al Mercado de Valores, sino también la necesaria incorporación en el Derecho Societario por cuanto involucra aspectos de constitución de sociedades, pluralidad de personas, Órganos de Administración y las principales operaciones de Reorganización Societaria como son las fusiones y escisiones.

Por tanto, al ver que hoy en día el mercado de constituciones de SPAC y su conexión con el mundo de las operaciones de Mergers \& Acquisition, hace que como ya lo hemos mencionamos líneas arriba, traer a la actualidad "recuerdos no tan gratos de la aplicación de los SPAC" de los años 90 , pero hoy en día con una serie de correcciones y protecciones adicionales, hace que en los últimos tres (03) años hayan convertido a las SPAC en una realidad muy apetecible que incluso el multimillonario ingles Richard Branson ${ }^{4}$, dueño de la compañía Virgin Galactic, la cual justamente salió a cotizar a la Bolsa de Valores de Nueva York a través de una empresa con propósito especial de adquisición (SPAC) siendo considerada dicha operación como un caso de éxito.

En conclusión, siendo bastante conservadores podemos definir a las empresas con propósito especial de adquisición (special purpose acquisition company) - sociedades SPAC, denominada también vehículo de inversión o compañías de cheque en blanco (blank check companies), como aquellas sociedades cotizadas sin actividad regular (salen a Bolsa sin activos) y que se constituyen con un mínimo tanto de personas como también lo puede ser de capital, en donde a través de dicha empresa recaudarán capital realizando una Oferta Pública de Venta (OPV) o también denominado Oferta Pública Inicial (IPO), con el objetivo de utilizar dicho capital (el mismo que estará en un patrimonio en fideicomiso y a cambio se les darán acciones y/o warrants) para adquirir la totalidad o una participación de otra sociedad sea cotizada o no cotizada a través de una compraventa de acciones, fusión, escisión o cualquier otra forma de operación permitida por la normativa, y para que la empresa privada adquirida bajo cualquiera de las modalidades citadas, pase a cotizar en bolsa al menos de manera indirecta ya que va a sustituir a la SPAC, con lo cual se convierte en un mecanismo rápido y más barato de ingreso al mundo bursátil. Es decir, las SPAC buscan vender una propuesta de inversión.

\section{4. ¿CUÁLES SON LOS RASGOS CARACTERÍSTICOS DE LAS SPAC?.}

La doctrina no ha manifestado una posición firme al respecto, sin embargo, en base a nuestra experiencia y en virtud a lo ya señalado líneas arriba, podemos mencionar a nuestra consideración algunos rasgos que se aplican de manera universal para estos tipos de empresas:

a) Inversión mínima, es importante fijar una inversión mínima, esto para mostrar a los futuros inversionistas que esta empresa se encuentra garantizada al menos con un mínimo de capital.

b) Pluralidad de personas, creemos que podría coexistir con la figura de las sociedades anónimas abiertas para el caso peruano, en cuanto a su modelo de formas de constituir, o en todo caso fijar un mínimo más accesible para este tipo de empresas.

4 En Julio del 2021 realizó el primer viaje con pasajeros de su empresa de turismo espacial Virgin Galactic 
c) Debe ser cotizada, necesariamente deben ser sociedades cotizadas, ya que a través de ella se proyectará el OPV o IPO para levantamiento de capital.

d) Debe de contar con una solicitud de admisión a negociación dada por la entidad supervisora en materia de mercado de valores, en nuestro caso por la Superintendencia de Mercado de Valores (SMV).

e) Debe ser utilizada solo para operaciones de fusiones y adquisiciones (M\&A), o cualquier otra modalidad que admita este tipo de operaciones.

f) Estas operaciones deben de contar con una regulación tributaria acorde para dichas operaciones (al menos darles facilidades).

g) Buscan ingreso a bolsa las sociedades privadas adquiridas a través de cualesquiera de las operaciones de M\&A de una manera más rápida y menos costosa.

h) Van a estar supervisadas por las Instituciones supervisoras y fiscalizadoras en materia de Mercado de Valores.

i) Deben de contar con un plazo determinado para realizar las operaciones de M\&A (algunas normativas extranjeras suelen regular hasta 24 meses de duración para realizar dichas operaciones).

j) Deben de contar con un derecho de separación y acceso al reembolso, esto en el caso que la operación de fusión, adquisición o cualquiera otro relacionado a estos les puedan perjudicar a los socios (principalmente minoritarios).

k) Fondo fideicomiso, el capital que se recaude deberá estar en un fideicomiso con el propósito de ser utilizado para la fusión u adquisición de una empresa.

I) Preparar los nuevos estatutos, ya que las SPAC deben de adoptar fuertes medidas respecto a las políticas de buen gobierno corporativo, esto con el propósito de alinear las buenas iniciativas entre los inversores, gestores y las instituciones participantes en el mercado donde el SPAC va actuar.

\section{5. ¿QUÉ ES LA OFERTA PÚBLICA DE VENTA (OPV) U OFERTA PÚBLICA INICIAL (IPO)? Y QUE SEÑALA NUESTRA REGULACIÓN DE MERCADO DE VALORES.}

Como hemos señalado para que las SPAC ejecuten sus tareas de adquisiciones, es que la OPV o IPO (o también se les conocen como OPS u Oferta Pública de Suscripción) cobran mucho interés, ya que, con dichos fondos depositados en un patrimonio en fideicomiso, se podrán realizar dichas operaciones (para el presente paper nos estamos centrando en la fusión).

Por ello, en mercados altamente desarrollados, una de las formas que tienen los fondos de inversión (investment funds or private equity) para la salida de sus inversiones es a través de las ofertas públicas iniciales de acciones, también conocidas como IPO (initial public offering). Es decir, listar las acciones de la compañía en un mercado centralizado de negociación de capitales y realizar una oferta primaria o, alternativa o conjuntamente secundaria a fin de permitir la venta por el fondo de inversión (Espinoza, 2015 ).

Por otro lado, también la Oferta Pública de Venta es la invitación que un propietario de valores inscritos en la bolsa de valores (acciones, bonos, etcétera) dirige al pública para venderlos. Este tipo de operaciones solo pueden ser realizadas por los inversionistas tenedores o titulares (propietarios) de valores que deseen transferirlos a otros inversionistas, recibiendo como contraprestación dinero, haciendo líquida su inversión (Rocca, 2017 ).

Respecto a esto, la Ley de Mercado de Valores peruano - Decreto Legislativo $N^{\circ} 861$ y sus modificatorias, señala al respecto: 


\section{Artículo 65.- Oferta Pública de Venta}

Constituye oferta pública de venta aquella que efectúan una o más personas naturales o jurídicas con el objeto de transferir al público en general o a determinados segmentos de éste, valores previamente emitidos y adquiridos.

Como vemos esta salida a Bolsa a través de la forma más común como lo puede ser el mercado primario, realizando la OPV/IPO/OPS, no hace más que ir perfeccionando la connotación que tiene una SPAC al usar este mecanismo para levantar capital y así comenzar a realizar su actividad de fusión o adquisición.

\section{UN POSIBLE RÉGIMEN JURÍDICO PARA LAS SPAC EN LA NORMATIVA PERUANA.}

¿Es posible un régimen jurídico para la SPAC en Perú?, la respuesta es sí. Si bien hemos afirmado que este tipo de modelo se aplica muy bien en mercado de capitales altamente desarrollados como los casos de EEUU y Holanda, esto no deja de ser atractivo también de poder desarrollarlo en el mercado peruano, a fin de dar a los inversionistas (sobre todo a los pequeños, ver el caso peruano en $E E U U^{5}$ ) la posibilidad de tener un apalancamiento de capital para así desarrollar sus planes de fusiones y/o adquisiciones en el futuro.

Contamos con una regulación de Mercado de Valores aún atractivo, pero que con la incursión de esta figura se tendría que actualizar dicha normativa para dar pie a que sea utilizado y así dar un mayor repunte al mercado de control de empresas en nuestro país mediante la atracción de inversionistas extranjeros y así dinamizar la economía del país, así como también obteniendo aspectos positivos en cuanto al ámbito tributario y de protección a las inversiones extranjeras (ejemplo convenios de estabilidad jurídica).

Ahora, ¿Qué temas debería de abarcar una futura norma? A nuestro entender y viendo algunas operaciones realizadas en Europa, es que podemos llegar a conclusión que serían dos (02) las normas principalmente a ser modificadas: la Ley General de Sociedades y la Ley de Mercado de Valores, y los temas a enfocarse básicamente serían:

1. Para la constitución de las SPAC, hay que ver si es suficiente lo que nos señala la Ley General de Sociedades con relación a las modalidades de constitución de las sociedades anónimas abiertas, quizás podamos incorporar un acápite más direccionado especialmente para esta figura.

2. Ver el tema del derecho de separación en la Ley General de Sociedades, por cuanto pueda ver la posibilidad de que un grupo de inversionistas/accionistas no se sientan finalmente cómodo con la posible fusión de la SPAC, por lo que se debería incorporar esta causal.

3. Ver el tema del derecho de reembolso en la Ley General de Sociedades, esto en virtud a que producto del ejercicio del derecho de separación, se deberá de cumplir con el reembolso, dentro de un determinado plazo.

4. Ver el tema de la reducción de capital en la Ley General de Sociedades, en el caso de que las acciones sean reembolsadas esto de acuerdo al numeral 3.

5 El caso de Rose Hill, un peruano que levantó US\$143 millones en Nasdaq y que iniciará compra de empresas en Latam. Una SPAC cuyo modelo de fondo de inversión tomó auge en los últimos dos años, ahora se aproxima al Perú, y otros países de la región. Diario Gestión del 21 de octubre de 2021. 
5. Ver el tema en la Ley de Mercado de Valores de incorpora la obligación de las SPAC de abrir una cuenta en fideicomiso donde se genere intereses en favor de ellos inversionistas/ accionistas hasta el momento en que se consolide la operación de fusión y/o adquisición.

6. Otro tema en la Ley de Mercado de Valores, es justamente otorgar un timing a la SPAC a fin de realizar y consolidar la operación de fusión/adquisición o cualquier otra forma regulada por la normativa o si es privada (compraventa de acciones).

7. Creemos que un cambio de denominación social en cuanto a las siglas en la Ley General de Sociedades momentáneo sería importante para las SPAC a fin de diferenciarlas en el mercado ya que su único objetivo sería de realizar operaciones de M\&A. Ya luego (en el presente caso de la fusión), podemos realizar un cambio de denominación/siglas (esto en virtud a que se va a aprovechar la reestructuración societaria, el cambio de los estatutos, entre otros), con lo cual estaría consolidadas.

8. Creemos que el derecho oposición de los acreedores de la sociedad target, puedan también realizar dicho ejercicio, pero es muy complicado por cuanto si la sociedad desea ingresar a este tipo de operaciones con el SPAC, deberá de estar totalmente saneada en su relacionamiento con terceros.

9. Y, una vez que se consolide (en este caso la fusión) con la inscripción en Registros Públicos y extinguida la sociedad, entonces dejará de ser aplicado la regulación especial para las SPAC tanto en la Ley General de Sociedades como en la Ley de Mercado de Valores, respectivamente.

10. El deber de divulgación de la información, el cual se tendría que incorporar alguna reforma a la Ley de Mercado de Valores sobre este tema (es decir que características debería tener dicha información que proveerá las SPAC), o quizás en la regulación sobre Hechos de Importancia, u otras Directivas que así lo señale la SMV, ya que como hemos mencionado estas SPAC figuran como una especie de "sociedad carcasa".

11. $\mathrm{Y}$, otras que podamos ir viendo en el camino que lo dejamos a los interesados por esta figura en estudiar, debatir y ver su necesidad de incorporar.

\section{EL PROCESO DE FUSIÓN CON LA SOCIEDAD CON PROPÓSITO ESPECIAL DE ADQUISICIÓN (SPAC).}

Luego, de haber explicado de manera general en cuanto a lo que se entiende por fusiones (propias e inversas), los diversos conceptos respecto a las SPAC, algunos de sus principales rasgos característicos, la OPV/IPO/OPS mediante el cual entran a captar los fondos y tener a las SPAC como una posible inclusión en nuestra regulación nacional, es que ahora vamos a conocer un poco de cómo se podría implementar un proceso de fusión, así como los diversos posibles actores involucrados en dichas operaciones.

Como ya hemos señalado, las SPAC agilizan mucho el ingreso de empresas (y por consiguientes sus accionistas) al mercado de valores, evitando la tediosa y a veces complicado cumplimiento de los requisitos regulatorios implementados en cada país.

En ese sentido, creemos que si las SPAC han cumplido de manera previa (y más aun siendo una sociedad carcasa) tener una ventaja en cuanto a su aplicación, es que la manera de poder realizar la operación de fusión creemos que tendría que realizarse de acuerdo a los siguientes pasos: 


\section{FASE 1 - ACUERDOS PREVIOS:}

1. Elegir la SPAC y/o la empresa target a fusionarse.

2. Negociar una Carta de Intenciones o Term Sheet en donde se pueda reflejar las cuestiones fundamentales de la valoración, derechos de voto, derecho de separación, reembolso, inversión privada en una sociedad cotizada, entre otros.

3. Firmar los términos del acuerdo de confidencialidad/Non-Disclosure Agreement (NDA).

4. Suscrito la carta y el NDA, entran al juego los asesores y todo el equipo elegido por el SPAC y la empresa target, en donde deberán de realizar el due Diligence (Due) y negociar los documentos propios de la operación. No está demás en mencionar aquellos documentos que se tengan que firmar para la realización de la Due, tales como: Process letter, clean team agreement, protocolo virtual data room, entre otros. Todos esos documentos, deben tener en cuenta mucho los temas regulatorios (mercado de valores), societarios y tributarios.

5. Culminado la Due y habiendo también definido los términos de la integración (en el presente caso una fusión por absorción), se realizan toda la documentación de carácter societario y bajo las regulaciones de los países en donde se encuentren tanto la SPAC como de la sociedad target.

\section{FASE 2 - OBTENCIÓN DE DOCUMENTOS DE CARÁCTER REGULATORIO:}

6. Definida la estructura, se negocian los acuerdos para la combinación y para la inversión, entre otros.

7. Una vez firmados, se lleva a cabo el anuncio al mercado y la presentación ante la Autoridad máxima del país en materia de Supervisión de Mercado de Valores (en el caso peruano SMV y EEUU la SEC).

8. Se da inicio al proceso de autorización para la admisión a cotización de las acciones de la entidad resultante, denominado "registration statement", que permitirá a los inversores desinvertir conforme a sus compromisos de lock-up.

9. Obtención de la autorización.

\section{FASE 3 - ACUERDO DE FUSIÓN}

10. Los Directorios y/o Gerente General, se encargarán de aprobar el texto del proyecto de fusión, más toda la información señalada en fases 1 y 2 .

11. Procede a la convocatoria a Junta General de Accionistas (en adelante "Junta") de acuerdo a plazos señalados en sus regulaciones. Seguro para el caso peruano, en aplicación a lo señalado para las sociedades anónimas abiertas.

12. En la Junta se tendrá que definir: en que los inversores de la SPAC deberán aprobar formalmente la operación (esto caerá por su propio peso ya que los sponsors en todo momento han estado informando a los inversores del SPAC, quienes ya tienen conocimiento y lo mismo sucede con los socios de la sociedad target) y determinar si algún inversor quiere un reintegro de las aportaciones iniciales, esto es aplicación del derecho de separación y reembolso.

13. De ejecutar dicho derecho, se tendrá que cumplir con otorgar un plazo para el reembolso, así como también definir si habrá o no una reducción de capital en caso así lo decidan los socios de la SPAC.

14. Cumplir con las publicaciones del acuerdo de la Fusión y ver la administración de la SPAC mientras se fusiona (quizás no tomar decisiones de carácter significativo mientras se fusiona, entre otros.) 


\section{FASE 4 - INTEGRACIÓN Y TOMA DE CONTROL}

15. Realizar la formalización de la escritura pública de la fusión.

16. Ver los requerimientos de una empresa listada en un Exchange después de la Fusión.

17. Extinción de la sociedad en Registros.

18. Emisión de las correspondientes certificaciones de acciones y su registro en el registro público de mercado de valores.

19. Probable que el símbolo en bolsa de la empresa cambie del de la SPAC al de la sociedad fusionada, el valor de la participación respectiva y el valor de las acciones del accionista de la SPAC seguirán siendo el mismo una vez completada la fusión.

20. Finalmente, los socios de la sociedad target, se convertirán en los nuevos dueños, asumiendo estos (en la totalidad de las veces) del control de la sociedad cotizada (pero de seguro regulando con ello los nuevos estatutos, denominación, gobierno corporativo, entre otros).

Por tanto, a manera de resumen general, en el gráfico abajo podemos ver tres (03) momentos importantes de esta operación de fusión:

- Primero: un espónsor y una cantidad de inversores iniciales ponen capital y reciben a cambio acciones (para el caso de los inversores iniciales también warrants, es decir opciones sobre acciones a un precio menor).

- Segundo: los accionistas iniciales venden sus acciones y opciones, pero hay nuevos inversores (que pueden ser privados) acceden a comprar las acciones de la SPAC.

- Tercero: luego seguro de un acuerdo de JGAen donde aprueban la fusión, la SPAC se fusiona con la compañía target, y en consecuencia los accionistas del SPAC se quedarán con un porcentaje de la sociedad inferior al $50 \%$ habitualmente, mientras que los accionistas de la sociedad target pasan a obtener mayor al $50 \%$ de las acciones de la sociedad absorbente.

Figure 1: The SPAC Merger Process

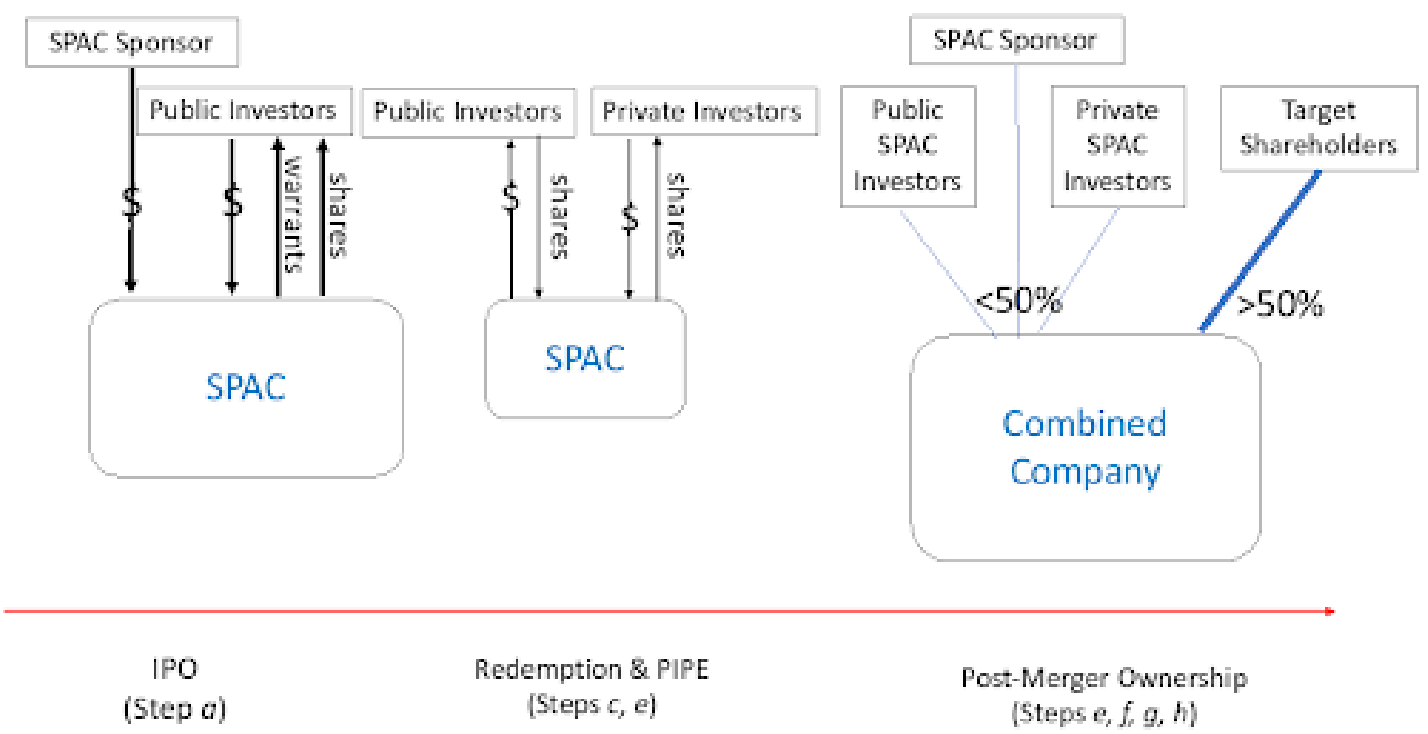

Fuente: A sober look at SPACS, Michael Klausner y Michael Olrogge. 
A manera de un off de record, viendo que son operaciones de M\&A las SPAC, hay algunas otras formas de operaciones un poco controversiales (claro las SPAC por una parte de la doctrina también los consideran así) que creemos sería materia de un análisis aún más exhaustivo y es acaso en que la figura de la asesoría técnica financiera podría coexistir con las SPAC.

Se sabe que la asesoría técnica financiera es una forma de adquisición poniendo en garantía los activos de la sociedad a adquirir (LBO, fusiones apalancadas, entre otros), si el propósito de las SPAC es para levantar capital y salir al mercado a realizar compras, creemos que con las fusiones apalancadas podría estar cubierto lo que desea la SPAC, pero, originaría quizás una operación apalancada mucho más compleja que la propia SPAC y sus operaciones de M\&A o también a la inversa por la serie de regulaciones que requieren un plan para eliminar la deuda, y solicitar a un experto para ver la vialidad de la operación. Entonces, si queremos forzar su coexistencia, esto ya será materia de otro análisis, pero lo dejamos en el tintero.

\section{CONCLUSIONES.}

1. Como hemos visto, las SPAC son operaciones cada vez más frecuentes y por tanto pueden dar seguridad por la cantidad de profesionales, agentes, instituciones comprometidas, y que toda ellas velarán porque se cumpla cada obligación que las SPAC hayan comunicado y adherido a lo señalado por la Institución de Mercado de Valores de cada país (en nuestro caso SMV).

2. Con el fin de dar una mayor operatividad a nuestro mercado de capitales, creemos que con esta figura existe un beneficio tanto que se activa el mercado de control de empresas, así como puede existir un ahorro de costos y una manera más inmediata a las sociedades target (accionistas) ingreso su ingreso a la Bolsa.

1. Las SPAC, es un vehículo jurídico de inversión con propósito exclusivo a ser utilizado en las operaciones de M\&A, lo que, al ser cotizado, se obligará a cumplir con toda la regulación en materia de mercado de valores, transparencia en la información, hechos de importancia, entre otros.

2. Al ser una sociedad carcasa, y si uno quiere participar de él, lo mínimo que se aconseja es investigar a los sponsor a fin de ver quiénes son, cuál es su perfil, su especialidad, ver su situación en el sistema financiero, ubicar sus actuaciones en la SMV, ver si cuentan con algunos antecedentes no gratos que hagan que los inversionistas se desistan, entre otros.

3. Como la asesoría técnica financiera aún no está muy bien utilizada en nuestro país, creemos que por la particularidad de estas operaciones SPAC, creemos que sería interesante analizar por la conveniencia de incorporar algunas modificaciones en nuestra Ley General de Sociedades, legislación Mercado de Valores, entre otras vinculadas a esta nueva figura para el derecho peruano.

4. Finalmente, creemos que hay un bajo nivel de riesgo para los inversores, ya que todos los fondos se mantienen en una cuenta fiduciaria hasta que se materializa la fusión y/o adquisición, y si no se produce, el dinero se devuelve a los inversores con sus intereses. $Y$ la inversión tiene un plazo fijo y no indefinido. 


\section{REFERENCIAS}

- Espinosa, L. (2015). Desinversiones por fondos de inversión o private equity. Fusiones \& Adquisiciones. Instituto Pacífico.

- Otaegui, Julio C. (1981). Fusión y Escisión de Sociedades Comerciales.Abaco.

- Rocca, L. (2017). El Mercado de Valores en fácil. PUC.

- De Haro, José Luis (2021). ¿Qué son las SPAC?, la nueva moda para salir a bolsa en EEUU. https://www.eleconomista.es/mercados-cotizaciones/noticias/10664507/07/20/Que-son-lasSPAC-la-nueva-moda-para-salir-a-bolsa-en-EEUU.html

- Baron, S. (2021). ¿Qué es una SPAC?. Características, ventajas y riesgos. https://www.finect. com/usuario/santiagobaron/articulos/que-spac-caracteristicas-ventajas-riesgos

- Ley N² 26887 - Ley General de Sociedades, publicado el 09 de diciembre de 1997.

- Ley $N^{\circ} 31112$ - Ley que establece el Control Previo de Operaciones de Concentración Empresarial, publicado el 07 de enero de 2021.

- Anteproyecto de la Ley de Mercado de Valores Español - mayo 2021.

Fecha de recepción : 26 de octubre de 2021

Fecha de aceptación : 18 de noviembre de 2021 